\title{
Magnetohydrodynamic mixed convection flow in vertical concentric annuli with time periodic boundary condition: Steady periodic regime
}

\author{
BASANT K JHA and BABATUNDE AINA* \\ Department of Mathematics, Ahmadu Bello University, Zaria, Nigeria \\ e-mail: basant777@yahoo.co.uk; ainavicdydx@gmail.com
}

MS received 4 December 2015; revised 15 April 2016; accepted 22 April 2016

\begin{abstract}
This work reports an analytical solution for fully developed mixed convection flow of viscous, incompressible, electrically conducting fluid in vertical concentric annuli under the influence of a transverse magnetic field, where the outer surface of inner cylinder is heated sinusoidally and the inner surface of outer cylinder is kept at a constant temperature. The analysis is carried out for fully developed parallel flow and steady-periodic regime. The governing dimensionless momentum and energy equations are separated into steady and periodic parts and solved analytically. Closed form solutions are expressed in terms of modified Bessel function of first and second kind. The influence of each governing parameters such as magnetic field parameter, Prandtl number and the dimensionless frequency of heating on flow formation and thermal behaviour are discussed with the aid of graphs. During the course of investigation, it is found that the oscillation amplitude of the friction factor is maximized at a resonance frequency near the surface of the concentric annuli where there is periodic heating. Furthermore, increasing transverse magnetic field decreases the oscillation amplitude of the friction factor.
\end{abstract}

Keywords. Mixed convection; transverse magnetic field; concentric annuli; periodic heating.

\section{Introduction}

The magnetohydrodynamic (MHD) phenomenon has received considerable attention during the last two decades due to its importance from the energy generation point of view, and one may envisage MHD generators for power generation. MHD pumps are already in use in chemical energy technology for pumping electrically conducting fluids at some of the atomic energy centre. Besides these applications, when the fluid is electrically conducting, the free convection flow is appreciably influenced by an imposed magnetic field. Williams in 1930 first started the use of electrically conducting fluid in pipes, who performed experiments using a copper sulphate solution measuring a dc voltage across the channel. Hartmaan and Lazarus [1] studied the MHD flow of an electrically conducting fluid in circular pipes under the action of transverse magnetic field. They used mercury as a conducting fluid. Shercliff $[2,3]$ made a detailed theoretical and experimental study for the flow of conducting fluid in pipes especially because of its utility in electromagnetic flow measurements. Hunt [4] presented an analysis of laminar motion of a conducting liquid in a rectangular duct with conducting walls in the presence of uniform transverse magnetic field. Tezer-Sezgin [5] investigated numerically the MHD flow of an

*For correspondence incompressible, viscous, electrically conducting fluid in a rectangular duct with an external uniform magnetic field employing boundary element method (BEM), considering the walls parallel to the applied magnetic field were conducting while the other two walls which were perpendicular to the field were insulators. Hosseinzadeh et al [6] studied numerically the MHD flow through a pipe of rectangular and circular cross sections having arbitrary conducting walls using the constant and the continuous linear boundary elements method (BEMs). Oreper and Szekely [7] analyzed the buoyancy driven flow in a rectangular cavity under the action of externally imposed magnetic field. Alboussiere et al [8] performed an asymptotic analysis to study the buoyancy driven convection in a uniform magnetic field. For closed geometry, Garandet et al [9] studied the problem of free convective flow in a rectangular enclosure in the presence of transverse magnetic field. Ghosh [10] studied asymptotic behaviour of transient MHD viscous flow in a revolving parallel-plate channel with oscillatory pressure gradient for large frequencies of oscillation at very small Ekman numbers. In recent articles, Sheikholeslamia et al [11] investigated the magnetic field effect on nanofluid flow and heat transfer in a semi-annulus enclosure via control volume based finite element method. Khan and Ellahi [12] studied the effects of magnetic field and porous medium on a unidirectional flow of a second grade fluid. Farhad et al [13] examined the slip effect on hydromagnetic rotating 
flow of viscous fluid through a porous space. Also, Farhad et al [14] investigated the effects of slip condition on the unsteady magnetohydrodynamics (MHD) flow of incompressible visco-elastic fluids in a porous channel under the influence of transverse magnetic field and Hall current with heat and mass transfer. Recently, Jha et al [15] carried out a study of combined influence of externally applied transverse magnetic field and suction/injection on steady natural convection flow of conducting fluid in a vertical microchannel. They reported that volume flow rate decreases with increase in Hartmann number. Jha et al [16] investigated steady natural convection flow of conducting fluid in a vertical parallel plate microchannel in the presence of transverse magnetic field. In another related article, Jha et al [17] conducted a theoretical investigation of steady fully developed MHD natural convection flow of conducting fluid in micro-concentricannuli in the presence of radial magnetic field. The fully developed MHD natural convection flow in a vertical annular microchannel was investigated by Jha et al [18]. Also, Jha and Aina [19] studied analytically the steady fully developed mixed convection flow of electrically conducting fluid in a vertical micro-annulus in the presence of transverse magnetic field. They discovered that an increase in magnetic field parameter leads to decrease in the rate of heat transfer at inner cylinder while it increases at outer cylinder.

On the other hand, heat transfer in channels or tubes with oscillatory surface temperature occurs in many industrial, engineering processes and natural phenomenon. Interest in this subject is due to its practical applications such as the automatic control systems, electrical and electronic component frequently subjected to periodic heating. Several studies have been conducted on the influence of periodic heating on flows. Amongst them are the works of Sparrow and Gregg [20] that considered a case in which the surface temperature varies slightly about a mean level, which is higher than the ambient temperature. Their studies were restricted to small amplitudes of the surface temperature variation. The same problem was solved by Chung and Anderson [21] using a slightly different perturbation expansion. There results were still restricted to small amplitudes. Yang et al [22] investigated the laminar natural convection flow with oscillatory surface temperature by using finite difference approach and were able to overcome the restrictions on the amplitude. Nanda and Sharma [23] were also able to circumvent the limitation of the result for only small amplitude by separating the temperature and velocity into steady and oscillatory components. Also, BarCohen and Rohsenow [24] conducted a study on fully developed convection between two periodically heated parallel plates, while Wang [25] studied the effect of Strouhal number on the development of boundary layers in a vertical channel whose boundary is subjected to periodic heating and periodic heat flux.
Resonance phenomenon was envisaged in the works of Lage and Bejan [26], Antohe and Lage [27] and Kwak et al [28] in which the heat flux through a vertical surface fluctuates with an amplitude that, for fixed values of the other parameters, reaches a maximum for a given value of angular frequency called the resonance frequency. They reported that a large amplitude wall temperature oscillation causes an increase in the time of average rate of heat transfer and that increase in maximum at a resonance frequency. Barletta and Zanchini [29] studied analytically the time periodic laminar mixed convection in an inclined channel with the temperature of one wall constant and the other wall a sinusoidal function of time and ignoring viscous dissipation. Their result showed that for every Prandtl number greater than 0.277 , there exists a resonance frequency that maximizes the amplitude of the friction factor oscillations at the unsteady temperature wall. In another related study, Barletta and Rossi di Schio [30] investigated the mixed convection flow in a vertical circular duct with time-periodic boundary conditions: steady periodic regime. They reported in their work that there exists resonance frequency for which the velocity oscillations reaches a maximum and this depends on the value assumed by the Prandtl number and the radial position. In addition, the resonance frequency which maximized the amplitude of the friction factor is a decreasing function of Prandtl number.

The objective of this study is to present a theoretical analysis on MHD mixed convection flow in a vertical concentric annuli under steady periodic regime provided with time periodic boundary conditions. The governing dimensionless momentum and energy equations are separated into steady and periodic parts and solved analytically. Closed form solutions are expressed in terms of modified Bessel function of first and second kind of order zero. These solutions generally deserve great attention, since the application of a magnetic field has been found to be effective tool in controlling the convection current. The current work is aimed as an extension of the previous analytical studies to provide some insight into a number of industrial applications, which use similar configurations.

\section{Mathematical analysis}

A fully developed mixed convection flow of viscous, incompressible and electrically conducting fluid in an annular gap between two vertical concentric cylinders of infinite length having a periodic variation of temperature with time is considered. Figure 1 displays the schematic diagram of the system examined and the co-ordinate axes. The velocities are assumed to be in a range such that flow is always laminar. The flow is assumed to be parallel so that the $X$-component $U$ of the velocity vector $\vec{U}$ is non-zero. Also, a uniform magnetic field $B_{0}$ is assumed to be acting perpendicular to the flow direction. We assume that the 


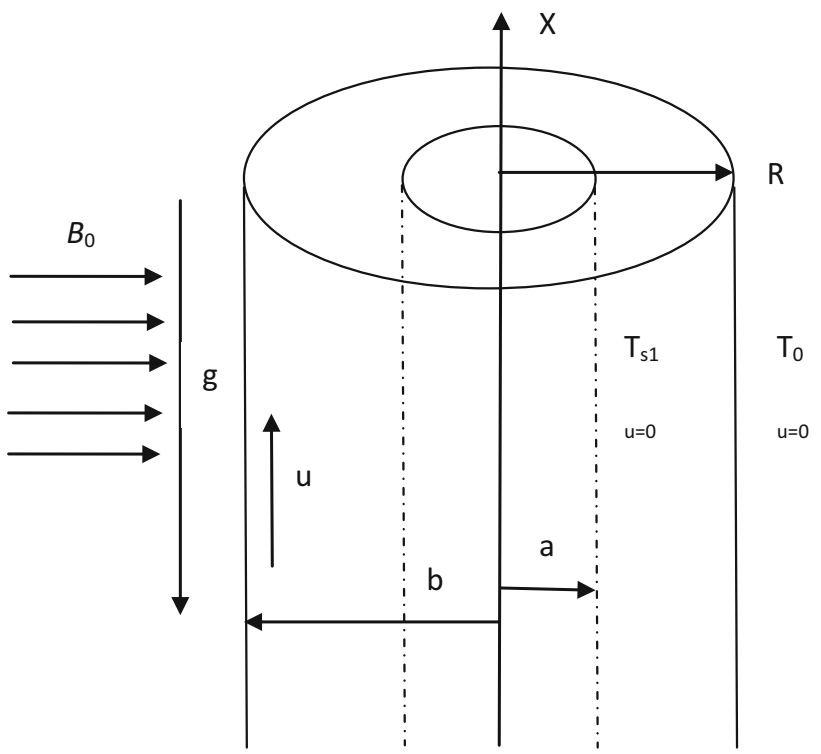

Figure 1. Flow configuration and coordinates system.

magnetic Reynolds number is very small, which corresponds to negligibly induced magnetic field compared to the externally applied one. Assuming a hydrodynamically and thermally fully developed flow, the continuity equation drops to $\partial U / \partial X=0$, i.e. $U=U(R, t)$. The surfaces of the cylinders forming the annulus are heated asymmetrically with the outer surface of inner cylinder heated sinusoidally while the inner surface of the outer cylinder is fixed at constant temperature. A pressure gradient is taken along the $X$-axis parallel to the axis of cylinders. The Boussinesq approximation is assumed to be valid. The outer surface of the inner cylinder $(R=a)$ is kept at an oscillating temperature with time, namely

$$
T(X, a, t)=T_{1}+\Delta T \cos (\omega t)
$$

It is assumed that the thermal boundary condition (1) does not yield any net fluid heating or cooling, heat transfer occurs only in the radial direction, so that

$$
\frac{\partial T}{\partial X}=0
$$

i.e. $T=T(R, t)$. The prescribed mass flow rate is assumed to be stationary, therefore average velocity in a cylinders cross section, defined as

$$
U_{0}=\frac{2}{b^{2}-a^{2}} \int_{a}^{b} R U(R, t) d R
$$

is time-independent. Assuming the equation of state $\rho=$ $\rho(T)$ is considered as linear,

$$
\rho=\rho_{0}\left[1-\beta\left(T-T_{0}\right)\right]
$$

where $T_{0}$ is the reference temperature with respect to both the annulus cross section and to a period of time namely

$$
T_{0}=\frac{\omega}{\pi\left(b^{2}-a^{2}\right)} \int_{0}^{\frac{2 \pi}{\omega}} d t \int_{a}^{b} R T(R, t) d R
$$

Since $\frac{\partial T}{\partial X}=0, T_{0}$ is a constant.

The mathematical model employed herein is similar to the work discussed by Barletta and Rossi di Schio [30]. Barletta and Rossi di Schio [30] analysed the mixed convection flow in a vertical circular duct (tube) due to periodic heating of the tube surface. In the present work, we analysed the mixed convection flow of conducting fluid in a vertical concentric annuli formed by two infinite co-axial vertical cylinders in the presence of transverse magnetic field. Mixed convection flow inside the concentric annuli is generated due to periodic heating of outer surface of inner cylinder as well as constant pressure gradient in vertical direction. Using Boussinesq approximation, the governing momentum equation of conducting fluid in the presence of transfer magnetic field is as follows:

$$
\frac{\partial U}{\partial t}=\frac{1}{\rho_{0}} \frac{\partial P}{\partial X}+g \beta\left(T-T_{0}\right)+\frac{1}{\rho_{0}} \frac{\mu}{R} \frac{\partial}{\partial R}\left(R \frac{\partial U}{\partial R}\right)-\frac{\sigma B_{0}^{2} U}{\rho_{0}}
$$

where $P=p+\rho_{0} g X$ is the difference between the pressure and hydrostatic pressure. By differentiating both sides of Eq. (6) with respect to $X$, one obtains $\frac{\partial^{2} P}{\partial X^{2}}=0$. The result implies an existence of a function $A(t)$ such that

$$
\frac{\partial T}{\partial X}=A(t)
$$

Then, Eq. (6) can be rewritten as

$\frac{\partial U}{\partial t}=\frac{1}{\rho_{0}} A(t)+g \beta\left(T-T_{0}\right)+\frac{1}{\rho_{0}} \frac{\mu}{R} \frac{\partial}{\partial R}\left(R \frac{\partial U}{\partial R}\right)-\frac{\sigma B_{0}^{2} U}{\rho_{0}}$.

The energy balance equation is given by

$$
\frac{\partial T}{\partial t}=\frac{\alpha}{R} \frac{\partial}{\partial R}\left(R \frac{\partial T}{\partial R}\right) \text {. }
$$

The non-dimensional quantities in the above equation are defined as

$$
\begin{aligned}
& \theta=\frac{T-T_{0}}{\Delta T}, \quad r=\frac{R}{a}, \quad d=\frac{b}{a}, \quad u=\frac{U}{U_{0}}, \quad \eta=\omega t, \\
& \Omega=\frac{a^{2} \omega}{v}, \quad \lambda=\frac{a^{2} A(t)}{\mu U_{0}}, \quad \operatorname{Re}=\frac{a U_{0}}{v}, \\
& G r=\frac{g \beta \Delta T a^{3}}{v^{2}}, \quad \xi=\frac{T_{1}-T_{0}}{\Delta T}, \quad \operatorname{Pr}=\frac{v}{\alpha}, \quad M^{2}=\frac{\sigma B_{0}^{2} a^{2}}{\mu}
\end{aligned}
$$


The physical quantities used in the above equations are defined in the nomenclature.

Substituting the dimensionless quantities defined in Eq. (10), the dimensionless momentum and energy equations are

$$
\begin{gathered}
\Omega \frac{\partial u}{\partial \eta}=\lambda+\frac{G r}{\operatorname{Re}} \theta+\frac{1}{r} \frac{\partial}{\partial r}\left(r \frac{\partial u}{\partial r}\right)-M^{2} u \\
\operatorname{Pr} \Omega \frac{\partial \theta}{\partial \eta}=\frac{1}{r} \frac{\partial}{\partial r}\left(r \frac{\partial \theta}{\partial r}\right) .
\end{gathered}
$$

The dimensionless boundary conditions for the present physical situation are as follows:

$$
\begin{gathered}
u(1, \eta)=0, \quad u(d, \eta)=0 \\
\theta(1, \eta)=\xi+\cos (\eta), \quad \theta(d, \eta)=0 .
\end{gathered}
$$

From Eqs. (3) and (5), the following two constraint equations for the dimensionless velocity and temperature are respectively,

$$
\begin{aligned}
& \int_{1}^{d} r u(r, \eta) d r=\frac{d^{2}-1}{2} \\
& \int_{0}^{2 \pi} d \eta \int_{1}^{d} r \theta(r, \eta) d r=0 .
\end{aligned}
$$

By differentiating with respect to $\eta$ both sides of the integral constraint on $u(r, \eta)$ expressed in Eq. (15), we have

$$
\int_{1}^{d} r \frac{\partial u(r, \eta)}{\partial \eta} d r=0
$$

\section{Analytical solution: velocity and temperature distribution}

In the steady periodic regime, the momentum and energy balance Eqs. (11) and (12), together with the boundary conditions (13) and (14) and the constraints (15) and (16) can be solved analytically by considering the function $u(r, \eta), \theta(r, \eta)$ and $\lambda(\eta)$ as the real parts of three complex valued functions, namely

$$
\begin{aligned}
& u(r, \eta)=\Re \mathrm{e}\left[u^{*}(r, \eta)\right] \\
& \theta(r, \eta)=\Re \mathrm{e}\left[\theta^{*}(r, \eta)\right] \\
& \lambda(\eta)=\Re \mathrm{e}\left[\lambda^{*}(\eta)\right] .
\end{aligned}
$$

On the account of Eqs. (11)-(16), the complex valued functions $u^{*}(r, \eta), \theta^{*}(r, \eta)$ and $\lambda^{*}(\eta)$ must be solution of the boundary value problem:

$$
\begin{gathered}
\Omega \frac{\partial u^{*}}{\partial \eta}=\lambda^{*}+\frac{G r}{\operatorname{Re}} \theta^{*}+\frac{1}{r} \frac{\partial}{\partial r}\left(r \frac{\partial u^{*}}{\partial r}\right)-M^{2} u^{*} \\
\operatorname{Pr} \Omega \frac{\partial \theta^{*}}{\partial \eta}=\frac{1}{r} \frac{\partial}{\partial r}\left(r \frac{\partial \theta^{*}}{\partial r}\right) \\
u^{*}(1, \eta)=0, \quad u^{*}(d, \eta)=0 \\
\theta^{*}(1, \eta)=\xi+\cos (\eta), \quad \theta^{*}(d, \eta)=0
\end{gathered}
$$

Therefore, one has

$$
\begin{aligned}
& u^{*}(r, \eta)=u_{a}^{*}(r)+\frac{G r}{\operatorname{Re}} u_{b}^{*}(r) \exp (i \eta) \\
& \theta^{*}(r, \eta)=\theta_{a}^{*}(r)+\theta_{b}^{*}(r) \exp (i \eta) \\
& \lambda^{*}(\eta)=\lambda_{a}^{*}+\frac{G r}{\operatorname{Re}} \lambda_{b}^{*} \exp (i \eta) .
\end{aligned}
$$

By substituting Eq. (23) into Eqs. (19)-(22), one obtains two independent boundary value problems.

The first boundary value problem is expressed as

$$
\begin{gathered}
\frac{1}{r} \frac{d}{d r}\left(r \frac{d u_{a}^{*}}{d r}\right)-M^{2} u_{a}^{*}+\lambda_{a}^{*}+\frac{G r}{\operatorname{Re}} \theta_{a}^{*}=0 \\
\frac{1}{r} \frac{d}{d r}\left(r \frac{d \theta_{a}^{*}}{d r}\right)=0 \\
u_{a}^{*}(1)=0, \quad u_{a}^{*}(d)=0, \\
\theta_{a}^{*}(1)=\xi, \quad \theta_{a}^{*}(d)=0 \\
\int_{1}^{d} r u_{a}^{*}(r) d r=\frac{d^{2}-1}{2} \\
\int_{1}^{d} r \theta_{a}^{*}(r) d r=0 .
\end{gathered}
$$

While the second is given by

$$
\begin{gathered}
\frac{1}{r} \frac{d}{d r}\left(r \frac{d u_{b}^{*}}{d r}\right)-M^{2} u_{b}^{*}-i \Omega u_{b}^{*}+\lambda_{b}^{*}+\theta_{b}^{*}=0 \\
\frac{1}{r} \frac{d}{d r}\left(r \frac{d \theta_{b}^{*}}{d r}\right)-i \Omega \operatorname{Pr} \theta_{b}^{*}=0 \\
u_{b}^{*}(1)=0, \quad u_{b}^{*}(d)=0, \\
\theta_{b}^{*}(1)=1, \quad \theta_{b}^{*}(d)=0, \\
\int_{1}^{d} r u_{a}^{*}(r) d r=0 .
\end{gathered}
$$

By employing the constraint on $\theta_{a}^{*}(r)$ yields $\xi=0$, i.e., $T=T_{0}$ 
The solution of Eqs. (24) and (25) under the conditions Eqs. (26)-(29) are

$$
\begin{gathered}
\theta_{a}^{*}(r)=0 \\
u_{a}^{*}(r)=C_{1} I_{0}\left(D_{1} r\right)+C_{2} K_{0}\left(D_{1} r\right)+\frac{\lambda_{a}^{*}}{M^{2}} . \\
\lambda_{a}^{*}=\frac{\left[d^{2}-1\right] M^{2}}{\left[\left(d^{2}-1\right)+2 Z_{4} M^{2}\right]} .
\end{gathered}
$$

While the solution of Eqs. (30) and (31) under the conditions Eqs. (32)-(34) are

$$
\begin{aligned}
& \theta_{b}^{*}(r)= C_{3} I_{0}\left(r D_{2}\right)+C_{4} K_{0}\left(r D_{2}\right) \\
& u_{b}^{*}(r)= C_{5} I_{0}\left(r D_{3}\right)+C_{6} K_{0}\left(r D_{3}\right)+\frac{\lambda_{b}^{*}}{i \Omega} \\
&+\frac{\left[C_{3} I_{0}\left(r D_{2}\right)+C_{4} K_{0}\left(r D_{2}\right)\right]}{\left[D_{3}^{2}-D_{2}^{2}\right]} \\
& \lambda_{b}^{*}=\frac{\left[Z_{8} Z_{12}-Z_{10} Z_{11}-Z_{16}\right] 2 i \Omega}{\left[\left(Z_{9} Z_{11}-Z_{7} Z_{12}\right) 2 i \Omega+\left(d^{2}-1\right)\right]}
\end{aligned}
$$

where $D_{1}=M, D_{2}=\sqrt{i \Omega \operatorname{Pr}}$, and $D_{3}=\sqrt{M^{2}+i \Omega}$.

The values of $C_{1}, \ldots, C_{6}$ and $Z_{1}, \ldots, Z_{16}$ are defined in the Appendix.

The fanning friction factor can be written as

$$
f \operatorname{Re}=\Re \mathrm{e}\left[f_{a}^{*} \operatorname{Re}+f_{b}^{*} \frac{G r}{\operatorname{Re}} \exp (i \eta)\right]
$$

where $f_{a}^{*}$ and $f_{b}^{*}$ are respectively given by

$$
\begin{aligned}
& f_{a}^{*} \operatorname{Re}=-\left.2 \frac{d u_{a}^{*}}{d r}\right|_{r=1} \\
& f_{b}^{*} \operatorname{Re}=-\left.2 \frac{d u_{b}^{*}}{d r}\right|_{r=1} .
\end{aligned}
$$

\section{Results and discussion}

In this study, the interactive effects of transverse magnetic field parameter $(M)$, Prandtl number $(\operatorname{Pr})$, and the dimensionless frequency $(\Omega)$ on flow formation and thermal behaviour are investigated. To examine the influence of these controlling parameters, the variations of dimensionless temperature, velocity, pressure drop, and friction factor are presented in figures 2-14.

Figure 2 illustrates the effects of the dimensionless frequency $(\Omega)$ and Prandtl number $(\operatorname{Pr})$ on the radial distribution of $\left|\theta_{b}^{*}\right|$. It is evident from figure 2 that $\left|\theta_{b}^{*}\right|$ is a monotonic increasing function of annular gap. This figure also shows that for $\Omega \leq 2$, the oscillation amplitude of the dimensionless temperature looks like a linear function
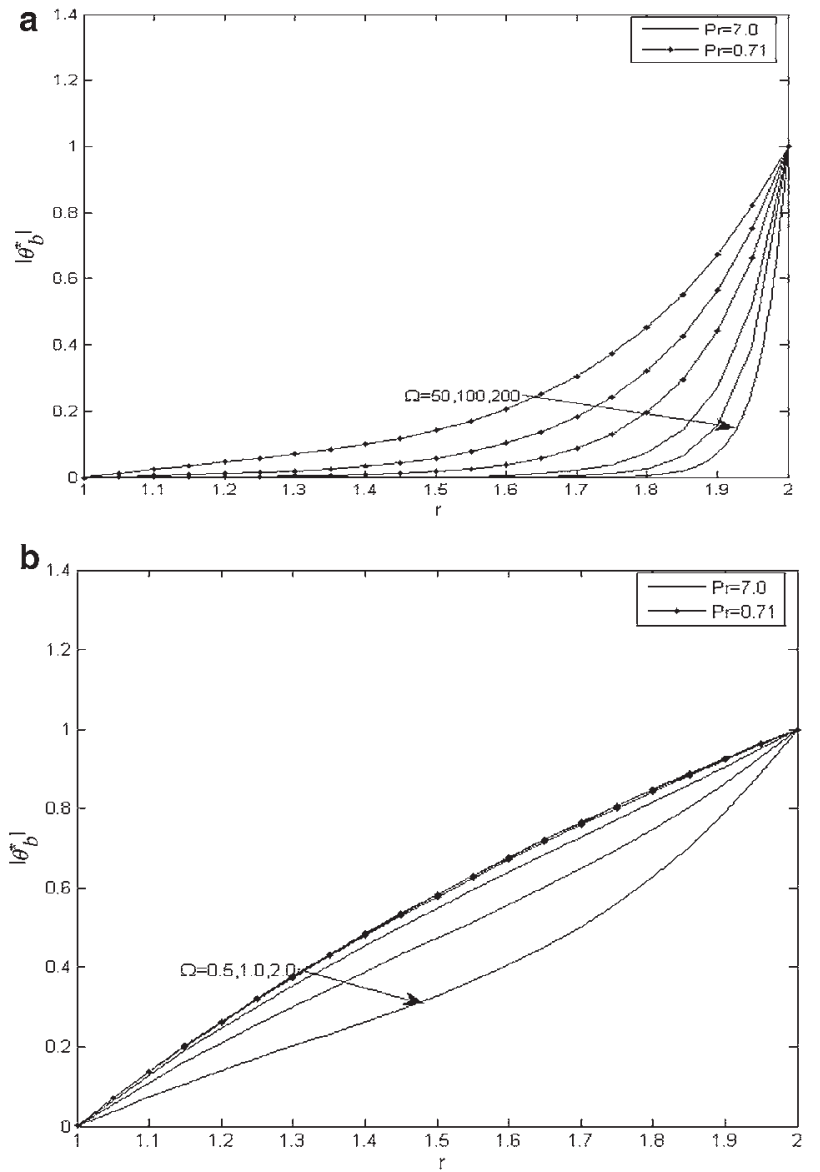

Figure 2. (a) Radial distribution of $\left|\theta_{b}^{*}\right|$ for different values of $\Omega$ (50< $\Omega<200$ ). (b) Radial distribution of $\left|\theta_{b}^{*}\right|$ for different values of $\Omega(0.5<\Omega<2)$.

of annular gap. Further, for large value of $\Omega \operatorname{Pr}$, the temperature oscillation tends to be sensible only in a narrow region of the concentric annuli surfaces.

Figures 3 and 4 exhibit the combined effects of dimensionless frequency $(\Omega)$ and Prandtl number $(\operatorname{Pr})$ on the radial distribution of $\left|u_{b}^{*}\right|$ for large value of dimensionless frequency $(50<\Omega<200)$ as well as small value of dimensionless frequency $(0.5<\Omega<2.0)$, respectively. It is observed that, increasing dimensionless frequency leads to decrease in $\left|u_{b}^{*}\right|$, as a result of reduction in intensity of the heating at outer surface of inner cylinder. By comparing figures 3 and 4, it is found that, a reduction in the Prandtl number $(\operatorname{Pr})$ results to a higher oscillation amplitude of the dimensionless velocity and this is because the thermal boundary layer that is being thickened which eventually results in an increase in $\left|u_{b}^{*}\right|$. Meanwhile, the oscillation amplitude of the dimensionless velocity has two local maxima, one close to the outer surface of inner cylinder and the other one at the inner surface of outer cylinder for both $\operatorname{Pr}=0.71$ and $\operatorname{Pr}=7$. Further, it is observed for smaller 


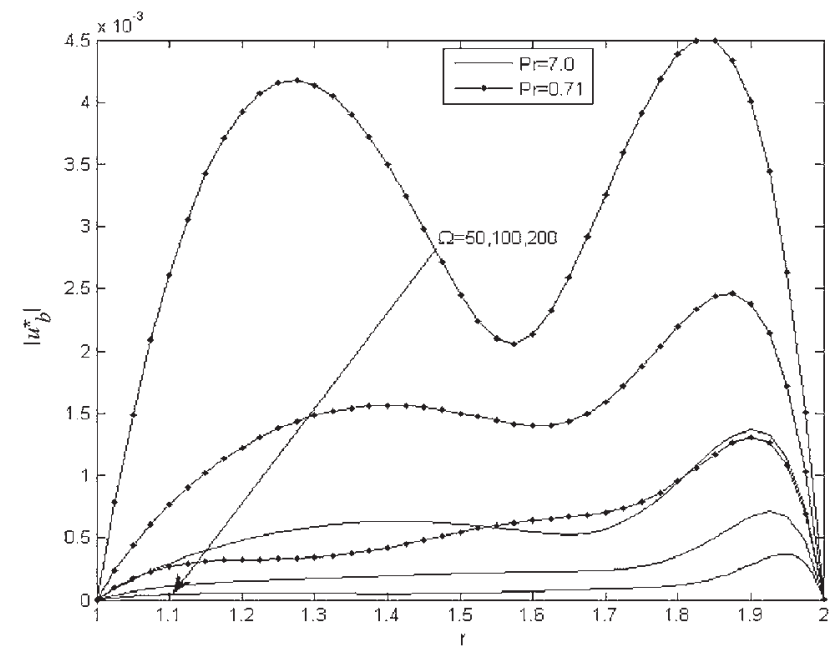

Figure 3. Radial distribution of $\left|u_{b}^{*}\right|$ for different values of $\Omega$ with fixed values of $M=0.5(50<\Omega<200)$.

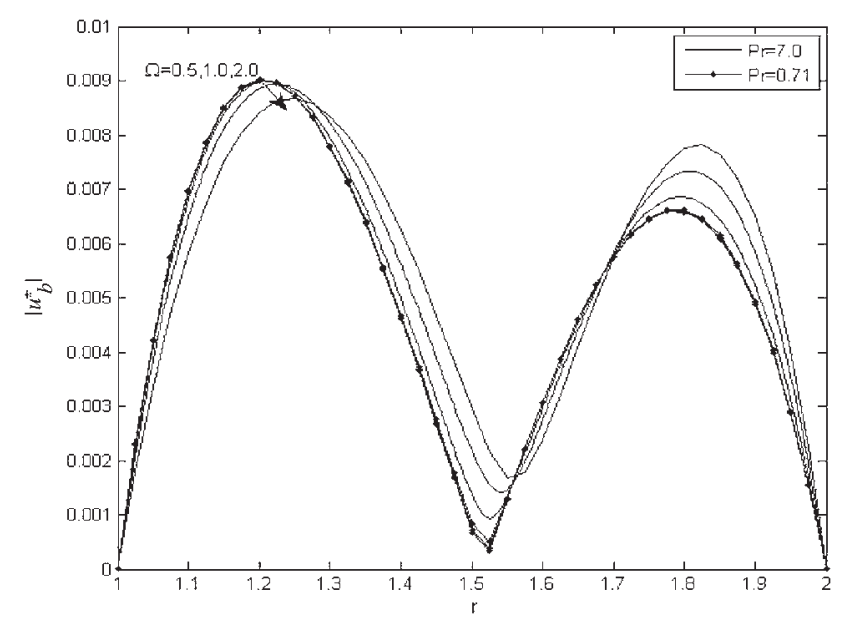

Figure 4. Radial distribution of $\left|u_{b}^{*}\right|$ for different values of $\Omega$ with fixed values of $M=0.5(0.5<\Omega<2.0)$.

dimensionless frequency $(0.5<\Omega<2.0)$, that the velocity gradient closer to the outer surface of inner cylinder that is heated periodically is higher compare to the inner surface of the outer cylinder; this reveals that the skin friction is higher on the outer surface of inner cylinder than the unheated surface of the annular. In addition, there also exist local minima approximately at the centre of the annulus signifying that the controlling parameter is not having much effect on the fluid velocity at that point and this is more prominent for smaller values of dimensionless frequency of the temperature oscillation $(0.5<\Omega<2.0)$.

Figures 5 and 6 illustrate the effects of magnetic field parameter $(M)$ and Prandtl number $(\operatorname{Pr})$ on the radial distribution of $\left|u_{b}^{*}\right|$ for large value of dimensionless frequency $(\Omega=50)$ and small value of dimensionless frequency

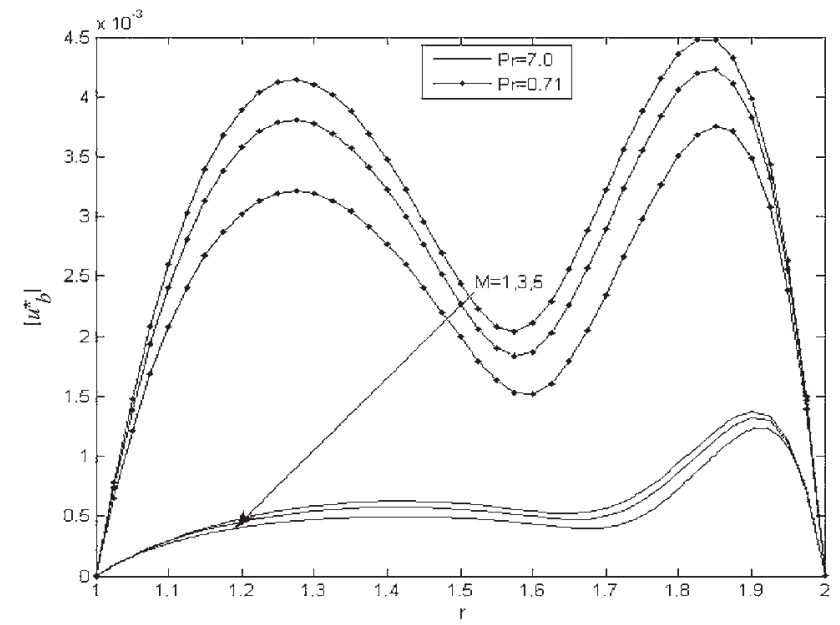

Figure 5. Radial distribution of $\left|u_{b}^{*}\right|$ for different values of $\mathrm{M}$ with fixed value of $\Omega=50$.

$(\Omega=0.5)$, respectively, with an increasing annular gap. By comparing figures 5 and 6 , it is evident that increasing the annular gap decreases the oscillation amplitude of the dimensionless velocity. It is also found that the radial distribution of $\left|u_{b}^{*}\right|$ decreases as magnetic field parameter $(M)$ increases. Physically speaking, the presence of transverse magnetic field sets in a resistive type force (Lorentz force), which is a retarding force on the velocity field. In addition, it is observed for both $\operatorname{Pr}=0.71$ and $\operatorname{Pr}=7$ that the oscillation amplitude of the dimensionless velocity has two local maxima both close to the annular surfaces. It is evident from figure 6 that there exists a point of stagnation in the flow domain, a point at which oscillation amplitude of the dimensionless velocity is almost zero at higher value of magnetic field. A comparison of figures 5 and 6 reveals that thermal diffusivity plays a decisive role in flow formation for high frequency of heating while the impact of thermal diffusivity is almost insignificant on flow formation for small frequency of heating.

Figures 7 and 8 depict the effects of Prandtl number $(\operatorname{Pr})$ and dimensionless frequency $(\Omega)$ on distribution of $\left|u_{b}^{*}\right|$ close to the annuli surfaces for $r=1.2$ and $r=1.8$, respectively. For fixed value of magnetic field parameter $(M)$ and Prandtl number $(\operatorname{Pr})$, it is evident that, at different radial positions, there exists a resonance frequency that gives a maximum oscillation amplitude of the dimensionless velocity and the resonance frequency decrease as the value of Prandtl number increase. In addition, it is evident that oscillation amplitude of the dimensionless velocity decreases monotonically with increase in Prandtl number $(\operatorname{Pr})$.

Figures 9 and 10 display the distribution of $\left|u_{b}^{*}\right|$ for different values of magnetic field parameter $(M)$ close to the annular surfaces for $r=1.2$ and $r=1.8$, respectively. It 




Figure 6. Radial distribution of $\left|u_{b}^{*}\right|$ for different values of $\mathrm{M}$ with fixed value of $\Omega=0.5$.

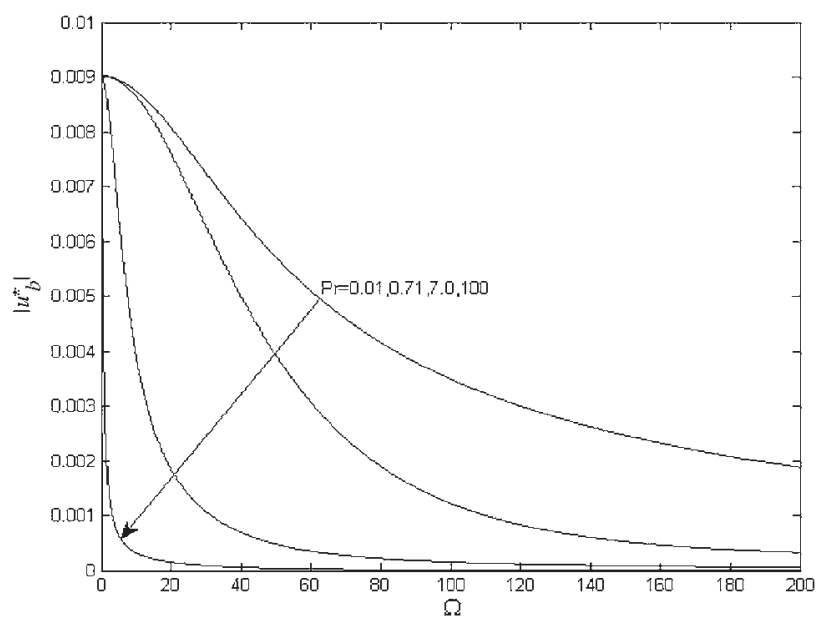

Figure 7. Distribution of $\left|u_{b}^{*}\right|$ versus $\Omega$ for different values of $\operatorname{Pr}$ with fixed value of $r=1.2, M=0.5$.

is evident that at different radial positions, there exists a resonance frequency that gives maximum oscillation amplitude of the dimensionless velocity. However, increasing magnetic field parameter $(M)$ leads to decrease in the fluid velocity in the concentric annuli.

Figure 11 shows the influence of Prandtl number (Pr) and dimensionless frequency $(\Omega)$ on the dimensionless pressure drop $\left|\lambda_{b}^{*}\right|$. It is observed that the oscillation amplitude of the dimensionless pressure drop $\left|\lambda_{b}^{*}\right|$ decreases monotonically with increase of Prandtl number and dimensionless frequency. This is due to the fact that as Prandtl number increases, thermal diffusivity of the fluid decreases which reduces the intensity of convection current. Also, higher value of dimensionless frequency is responsible for mild heating of the outer surface of inner cylinder.

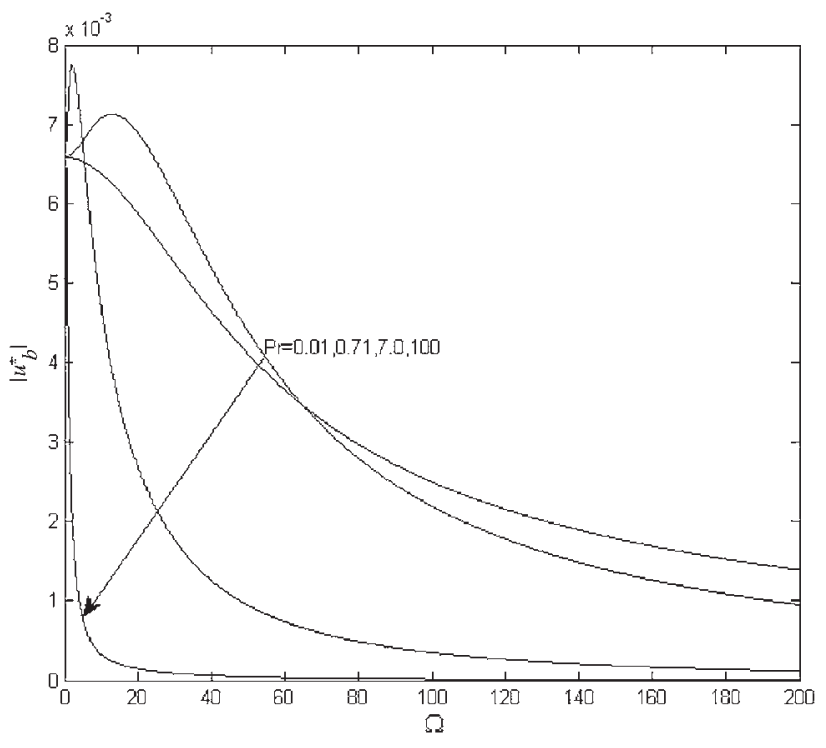

Figure 8. Distribution of $\left|u_{b}^{*}\right|$ versus $\Omega$ for different values of $\operatorname{Pr}$ with fixed value of $r=1.8, M=0.5$.

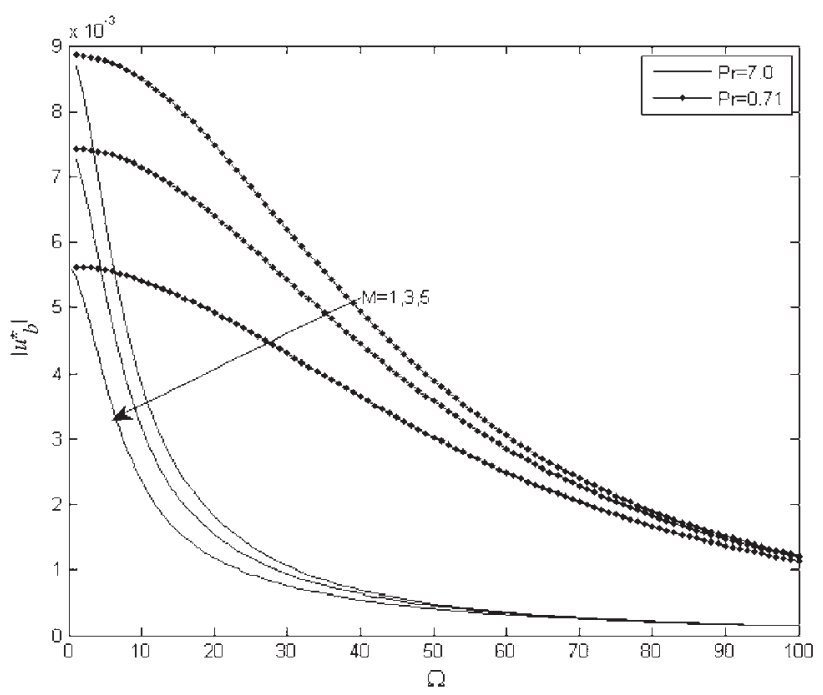

Figure 9. Distribution of $\left|u_{b}^{*}\right|$ versus $\Omega$ for different values of $\mathbf{M}$ with fixed value of $r=1.2$.

Figure 12 exhibits the effects of magnetic field parameter $(M)$ and dimensionless frequency $(\Omega)$ on the dimensionless pressure drop $\left|\lambda_{b}^{*}\right|$. It is evident that as magnetic field parameter $(M)$ increases, the dimensionless pressure drop $\left|\lambda_{b}^{*}\right|$ decrease. In addition, this figure shows that dimensionless pressure drop $\left|\lambda_{b}^{*}\right|$ is a monotonic decreasing function of dimensionless frequency $(\Omega)$ and that it decreases more rapidly for higher values of Prandtl number (Pr).

Figures 13 and 14 reported the oscillation amplitude of the friction factor versus dimensionless frequency $(\Omega)$ for different values of magnetic field parameter $(M)$ and 




Figure 10. Distribution of $\left|u_{b}^{*}\right|$ versus $\Omega$ for different values of $\mathrm{M}$ with fixed value of $r=1.8$.

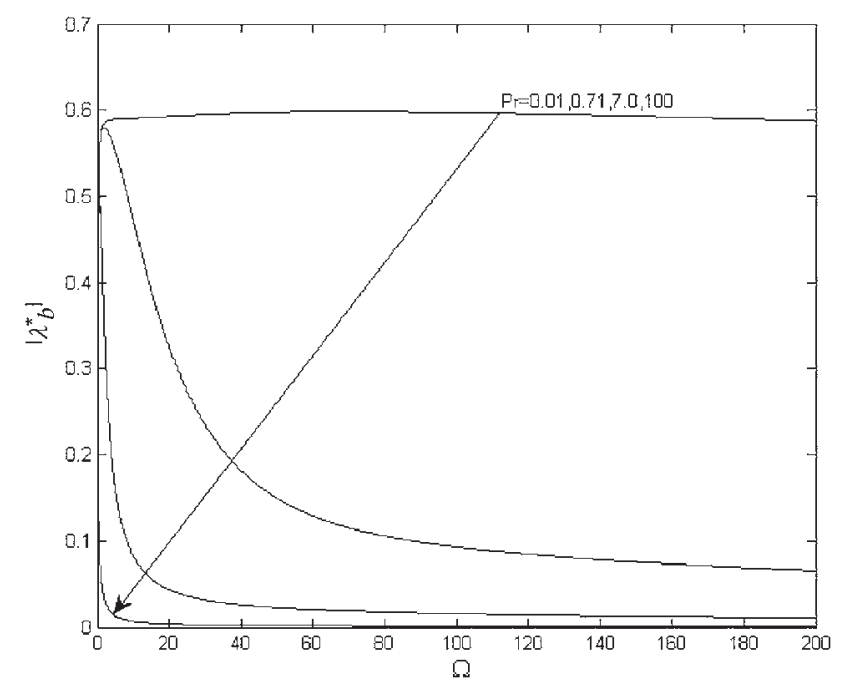

Figure 11. Distribution of $\left|\lambda_{b}^{*}\right|$ versus $\Omega$ for different values of Pr for $\mathrm{M}=0.5$.

Prandtl number $(\mathrm{Pr})$. It is noticed that there exists a resonance frequency for every assumed Prandtl number (Pr) and the resonance frequency is a monotonic decreasing function of the Prandtl number (Pr).

\section{Conclusions}

The mathematical model capturing the MHD mixed convection flow in a vertical concentric annuli due to periodic heating of one of the annular surfaces under relevant boundary and constraint equations is presented in dimensionless form. The expressions for velocity,



Figure 12. Distribution of $\left|\lambda_{b}^{*}\right|$ versus $\Omega$ for different values of $r$.

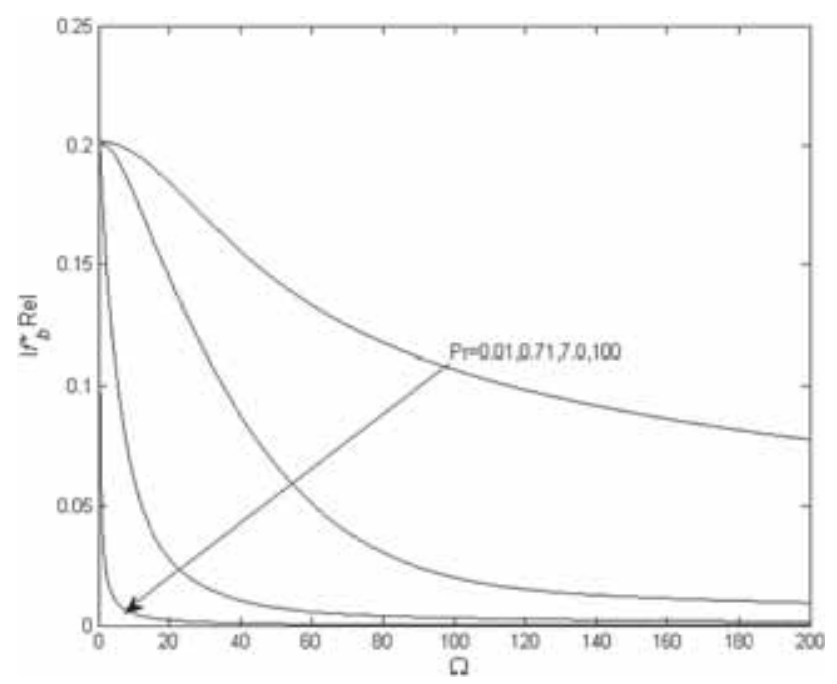

Figure 13. Distribution of $\left|\mathrm{f}_{\mathrm{b}}^{*} \operatorname{Re}\right|$ versus $\Omega$ for different values of $\operatorname{Pr}$ for $M=0.5$.

temperature and pressure drop are obtained. The effects of parameters controlling the present physical situation are discussed in detail. From the indicated results of the present problem, the following conclusions are made:

1. It is found that increasing magnetic field parameter decreases the oscillation amplitude of the dimensionless velocity.

2. The oscillation amplitude of the dimensionless velocity and temperature increases with increasing the gap between the concentric cylinders.

3. The oscillation amplitude of the dimensionless temperature, velocity and pressure drop is dependent on the frequency of heating, strength of transverse magnetic field and Prandtl number of the working fluid. 


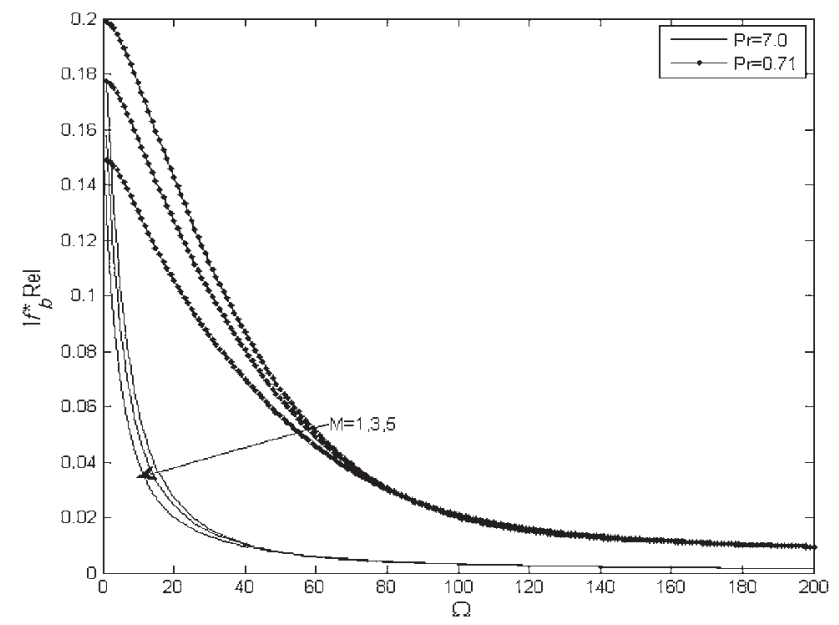

Figure 14. Distribution of $\left|\mathrm{f}_{\mathrm{b}}^{*} \operatorname{Re}\right|$ versus $\Omega$ for different values of $\mathrm{M}$.

4. It is found that, as transverse magnetic field parameter $(M)$ increases, the oscillation amplitude of the dimensionless pressure drop $\left|\lambda_{b}^{*}\right|$ decreases.

5. Finally, at different radial positions, there exists a resonance frequency that gives a maximum oscillation amplitude of the dimensionless velocity.

\section{Appendix}

$Z_{1}=K_{0}\left(D_{1}\right) I_{0}\left(D_{1} d\right)-K_{0}\left(D_{1} d\right) I_{0}\left(D_{1}\right)$,

$Z_{2}=\frac{\left[I_{0}\left(D_{1}\right)-I_{0}\left(D_{1} d\right)\right]}{Z_{1} M^{2}}, \quad Z_{3}=\frac{\left[K_{0}\left(D_{1} d\right)-K_{0}\left(D_{1}\right)\right]}{Z_{1} M^{2}}$,

$Z_{4}=\frac{\left[Z_{3}\left(d I_{1}\left(D_{1} d\right)-I_{1}\left(D_{1}\right)\right)-Z_{2}\left(d K_{1}\left(D_{1} d\right)-K_{1}\left(D_{1}\right)\right)\right]}{D_{1}}$,

$Z_{5}=K_{0}\left(D_{2} d\right) I_{0}\left(D_{2}\right)-K_{0}\left(D_{2}\right) I_{0}\left(D_{2} d\right)$,

$Z_{6}=K_{0}\left(D_{3}\right) I_{0}\left(D_{3} d\right)-K_{0}\left(D_{3} d\right) I_{0}\left(D_{3}\right)$,

$Z_{7}=\frac{\left[I_{0}\left(D_{3}\right)-I_{0}\left(D_{3} d\right)\right]}{Z_{6} i \Omega}, \quad Z_{8}=\frac{I_{0}\left(D_{3}\right)}{\left[D_{3}^{2}-D_{2}^{2}\right] Z_{6}}$,

$Z_{9}=\frac{\left[K_{0}\left(D_{3} d\right)-K_{0}\left(D_{3}\right)\right]}{Z_{6} i \Omega}, \quad Z_{10}=\frac{K_{0}\left(D_{3}\right)}{\left[D_{2}^{2}-D_{3}^{2}\right] Z_{6}}$,

$Z_{11}=\frac{\left[d I_{1}\left(D_{3} d\right)-I_{1}\left(D_{3}\right)\right]}{D_{3}}, Z_{12}=\frac{\left[d K_{1}\left(D_{3} d\right)-K_{1}\left(D_{3}\right)\right]}{D_{3}}$

$Z_{13}=\frac{C_{3}}{D_{2}}\left[d I_{1}\left(D_{2} d\right)-I_{1}\left(D_{2}\right)\right]$

$Z_{14}=\frac{C_{4}}{D_{2}}\left[d K_{1}\left(D_{2} d\right)-K_{1}\left(D_{2}\right)\right]$

$Z_{15}=\frac{1}{\left[D_{3}^{2}-D_{2}^{2}\right]}, \quad Z_{16}=Z_{15}\left[Z_{13}-Z_{14}\right]$,

$C_{2}=\lambda_{a} Z_{2}, \quad C_{3}=-\frac{K_{0}\left(D_{2}\right)}{Z_{5}}, \quad C_{4}=\frac{I_{0}\left(D_{2}\right)}{Z_{5}}$,

$C_{5}=Z_{9} \lambda_{b}+Z_{10}, \quad C_{6}=Z_{7} \lambda_{b}+Z_{8}$.

\section{Nomenclature}

$a$

$B_{0} \quad$ Constant magnetic flux density

$b \quad$ Radius of the outer cylinder

d Radius ratio

$f \quad$ Fanning friction factor

$g \quad$ Gravitational acceleration

Gr Grashof number

$i \quad$ Imaginary unit

$I_{n} \quad$ Modified Bessel function of first kind and order $n$

$K_{n} \quad$ Modified Bessel function of second kind and order $n$

Thermal conductivity

Integer number

Transverse magnetic field

p Pressure

$P \quad$ Difference between the pressure and the hydrostatic pressure

Pr Prandtl number

$R \quad$ Radial coordinate

Re Reynolds number

Re Real part of a complex number

$t$ Time

$T \quad$ Temperature

$T_{0} \quad$ Mean temperature in concentric annuli defined in Eq. (5)

$T_{1} \quad$ Mean temperature of the outer surface of inner cylinder

$u \quad$ Dimensionless velocity

$u^{*} \quad$ Dimensionless complex-valued function

$u_{a}^{*}, u_{b}^{*} \quad$ Dimensionless complex-valued function

$U \quad$ Fluid velocity

$X \quad$ Longitudinal coordinate in vertical direction

\section{Greek symbols}

$\alpha \quad$ Thermal diffusivity

$\beta \quad$ Volumetric coefficient of thermal expansion

$\Delta T \quad$ Amplitude of the temperature oscillations at the outer surface of inner cylinder

$\lambda \quad$ Dimensionless parameter defined in Eq. (10)

$\lambda^{*} \quad$ Dimensionless complex-valued function defined in Eq. (18)

$\lambda_{a}^{*}, \lambda_{b}^{*} \quad$ Dimensionless complex-valued function defined in Eq. (23)

$\eta \quad$ Dimensionless time

$\theta \quad$ Dimensionless temperature

$\theta_{a}^{*}, \theta_{b}^{*} \quad$ Dimensionless complex-valued function defined in Eq. (23)

$\mu \quad$ Dynamic viscosity

$v \quad$ Kinematic viscosity

$\Phi \quad$ Dimensionless heat flux

$\Phi_{a}^{*}, \Phi_{b}^{*} \quad$ Dimensionless complex-valued function 
$\rho \quad$ Mass density

$\rho_{0} \quad$ Mass density for $T=T_{0}$

$\omega \quad$ Frequency of the temperature oscillation

$\Omega \quad$ Dimensionless frequency

$\sigma \quad$ Electrical conductivity of the fluid

\section{References}

[1] Hartmaan J and Lazarus F 1937 Experimental investigations on the flow of mercury in a homogeneous magnetic field. $K$. Dan. Vidensk. Selsk. Mat. Fys. Medd 15(6): 1-45

[2] Shercliff J A 1953 Steady motion of conductingfluids in pipes under the action transverse magnetic field. Math. Proc. Camb. Philos. Soc. 49(1): 136-144

[3] Shercliff J A 1962 Magnetohydronamics pipe flow Part 2, high Hartmaan number. J. Fluid Mech. 13(4): 513-518

[4] Hunt J C R 1965 Magnetohydrodynamics flow in rectangular ducts. J. Fluid Mech. 21(4): 577-590

[5] Tezer-Sezgin M 1994 Boundary element methodsolutions of MHD flow in a rectangular duct. Int. J. Numer. Methods Fluids 18(10): 937-952

[6] Hosseinzadeh H, Dehghan M and Mirzaeib D 2012 The boundary elements method for Magneto-Hydrodynamics (MHD) Channel Flows at High Hartmaan Numbers, AMS classification: $65 \mathrm{~N} 38$

[7] Oreper G M and Szekely J 1983 The effect of an external imposed magnetic field on buoyancy driven flow in a rectangular cavity. J. Cryst Growth 64: 505-515

[8] Alboussiere T, Garandet J P, Moreau R 1983 Buoyancydriven convection with a uniform magnetic field, Part 1: Asymptotic analysis. J. Fluid Mech. 253: 545-563

[9] Garandet J P, Alboussiere T and Moreau R 1992 Buoy-ancy driven convection in a rectangular enclosure with a transverse magnetic field. Int. Commun. Heat Mass Transf. 35: 741-749

[10] Ghosh S K 1993 Unsteady hydromagnetic flow in a rotat-ing channel with oscillating pressure gradient. J. Phys. Soc. Jpn 62(11): 3893-3903

[11] Sheikholeslamia M, Gorji-Bandpy M, Ganji D D, Rana P and Soheil Soleimani 2014 Magnetohydrodynamic free convection of Al2O3-water nanofluid considering thermophoresis and Brownian motion effects. Comput. Fluid 94: 147-60

[12] Khan I and Ellahi R 2008 Some unsteady MHD flows of a second grade fluid through porous medium. J. Porous Media (ISI Indexed, I.F 0.684), 11(4): 389-400

[13] Farhad A, Norzieha M, SSharidan S, Khan I and Samiuthaq 2012a On hydromagnetic rotating flow in a porous medium with slip condition and hall current. Int. J. Phys. Sci. 7(10): 1540-1548
[14] Farhad Ali, Ilyas Khan, Samiulhaq, Norzieha Mustapha and Sharidan Shafie 2012b Unsteady magnetohydrodynamic oscillatory flow of viscoelastic fluids in a porous channel with heat and mass transfer. J. Phys. Soc. Jpn 81: 064402

[15] Basant K Jha, Babatunde Aina and A J Ajiya 2015 Role of suction/injection on MHD natural convection flow in a vertical micro-channel. Int. J. Energy Technol. 7(2): 30-39

[16] Basant K Jha, Babatunde Aina and Sani Isa 2015a MHD natural convection flow in a vertical micro-concentric-annuli in the presence of radial magnetic field: An exact solution. $J$. Ain Shams Eng. http://dx.doi.org/10.1016/j.asej.2015.07.010

[17] Basant K Jha, Babatunde Aina and Sani Isa 2015b Fully developed MHD natural convection flow in a vertical annular microchannel: An exact solution. J. King Saud Univ. (Sci.) 27: 253-259

[18] Basant K Jha, Babatunde Aina and Ajiya A J 2014 MHD natural convection flow in a vertical parallel plate microchannel. Ain Shams Eng. J. 6: 289-295

[19] Basant K Jha and Babatunde Aina 2015 MHD mixed convection flow in a vertical microannulus: An exact solution. Int. J. Fluid Mech. Res. 42(6): 537-552

[20] Sparrow E M and Gregg J L 1960 Newly quasi-steady freeconvection heat transfer in gases. J. Heat Mass Transf. Trans. ASME Ser. 82: 258-260

[21] Chung P M and Anderson A D 1961 Unsteady laminar free convection. ASME J. Heat Mass Transf. 83: 473-478

[22] Yang J W, Scaccia C and Goodman J 1974 Laminar natural convection about vertical plates with oscillatory surface temperature. Trans. ASME J. Heat Transf. 96: 9-14

[23] Nanda R J and Sharma V P 1963 Free convection laminar boundary layer in oscillatory flow. J. FluidMech. 15: 419-428

[24] Bar-Cohen A and Rohsenow W M 1984 Thermally optimum spacing of vertical natural convection cooled parallel plates. ASME J. Heat Transf. 106: 116-123

[25] Wang C Y 1988 Free convection between vertical plates with periodic heat input. ASME J. Heat Transf. 110: 508-511

[26] Lage J L and Bejan A 1993 The resonance of natural convection in an enclosure heated periodically from the side. Int. J. Heat Mass Transf. 36: 2027-2038

[27] Antohe B V and Lage J L 1996 Amplitude effect on convection induced by time periodic boundary conditions. Int J. Heat Mass Transf. 39: 1121-1133

[28] Kwak H S, Kvwahara J M, Hyun J M 1998 Resonant enhancement of natural convection heat transfer in a square enclosure. Int. J. Heat Mass Transf. 41: 2837-2846

[29] Barletta A and Zanchini E 2002 Time-periodic laminar mixed convection in an inclined channel. Int. J. Heat Mass Transf. 46: 551-563

[30] Barletta A and Rossi di Schio E 2004 Mixed convection flow in a vertical circular duct with time-periodic boundary conditions: Steady-periodic regime. Int. J. Heat Mass Transf. 47: 3187-3195 